\title{
Measurement of sound propagation in glass wool
}

\author{
Tarnow, Viggo
}

Published in:

Acoustical Society of America. Journal

Link to article, DOI:

10.1121/1.411952

Publication date:

1995

Document Version

Publisher's PDF, also known as Version of record

Link back to DTU Orbit

Citation (APA):

Tarnow, V. (1995). Measurement of sound propagation in glass wool. Acoustical Society of America. Journal, 97(4), 2272-2281. https://doi.org/10.1121/1.411952

\section{General rights}

Copyright and moral rights for the publications made accessible in the public portal are retained by the authors and/or other copyright owners and it is a condition of accessing publications that users recognise and abide by the legal requirements associated with these rights.

- Users may download and print one copy of any publication from the public portal for the purpose of private study or research.

- You may not further distribute the material or use it for any profit-making activity or commercial gain

- You may freely distribute the URL identifying the publication in the public portal

If you believe that this document breaches copyright please contact us providing details, and we will remove access to the work immediately and investigate your claim. 


\title{
Measurement of sound propagation in glass wool
}

Viggo Tarnow

Department of Applied Engineering Design and Production, DTU, Akademivej Bygning 358, DK 2800

Lyngby, Denmark

(Received 26 May 1994; accepted for publication 20 December 1994)

\begin{abstract}
A new acoustic method for directly measuring the flow resistance, and the compressibility of fibrous materials such as glass wool, is given. Measured results for monochromatic sound in glass wool are presented and compared with theoretically calculated results. The agreement between experimental results and theory is good. Results of measurements of characteristic impedance, attenuation, and phase shift for plane monochromatic traveling waves are presented and compared with theoretically calculated ones. Good agreement between experimental and theoretical results was found.
\end{abstract}

PACS numbers: 43.58. Bh, 43.20.Hq, 43.20.Jr, 43.55.Ev

\section{LIST OF SYMBOLS}

$a \quad$ radius of fibers

$b \quad$ square root of mean area per fiber

$C$ complex compressibility of air in glass wool

$C_{f} \quad$ complex compressibility function defined in tube

$C_{f}$ imaginary compressibility function. It equals $C_{i}$ inside sample, zero outside

$C_{f r}$ real compressibility function. It equals $C_{r}$ inside sample, $C_{0}$ outside

$C_{i} \quad$ imaginary part of compressibility of air in glass wool

$C_{r} \quad$ real part of compressibility of air in glass wool

$C_{0} \quad$ compressibility of air itself

$c_{p} \quad$ heat capacity per mass of air at constant pressure

$d$ dissipation factor for tube with glass wool

$d_{C} \quad$ dissipation factor due to imaginary part of compressibility of glass wool

$d_{R} \quad$ dissipation factor due to viscous resistance of glass wool

$d_{0} \quad$ dissipation factor for empty tube

$d \nu \quad$ element of volume

$d p \quad$ pressure increase

$d x \quad$ element of distance

$E \quad$ energy stored in resonator

$f \quad$ resonance frequency

$H_{0}^{1} \quad$ Hankel function of zero-order first kind

$H_{1}^{1} \quad$ Hankel function of first-order first kind

$k \quad$ sound pressure wave vector; real part is attenuation constant. Imaginary part is phase shift per length

$k_{t} \quad$ thermal wave vector

$L \quad$ length of tube from end plate to microphone

$l \quad$ length of sample

\section{INTRODUCTION}

This paper reports measurements of the material parameters compressibility and flow resistance that are used to describe the propagation of audible sound waves in glass wool. The method used to measure the compressibility and the resistance is new. It will be described in some detail.

Plane traveling waves can be described by attenuation per meter, phase shift per meter, and characteristic imped-
$P_{d} \quad$ power loss in resonator

$p \quad$ sound pressure

$p_{0}, p_{1} \quad$ sound pressure constants

$p(x) \quad$ pressure at point $x$

$R \quad$ flow resistance of glass wool-real

$R_{f} \quad$ resistance function; equals $R$ inside glass wool, zero outside

time

velocity

velocity constant

volume

space coordinate from end plate of tube to point in tube

$Z \quad$ characteristic impedance of air

$\gamma \quad$ heat capacity of air at constant pressure divided by heat capacity at constant volume: for air $=1.40$

$\Delta f \quad 3$-dB band with of resonance curve

$\eta \quad$ viscosity

$\kappa \quad$ coefficient of thermal conductivity of air

$\rho \quad$ effective mass density of air-real

$\rho_{g} \quad$ mass density of glass in fibers

$\rho_{w} \quad$ mass density of glass wool

$\rho_{0} \quad$ mass density of air itself

$\omega \quad$ cyclic frequency $=2 \pi \times$ frequency

$\omega_{c} \quad$ complex cyclic resonance frequency for tube with sample

$\omega_{r}$ real cyclic resonance frequency for tube with sample

$\omega_{0} \quad$ real cyclic resonance frequency for tube without sample

$\left\langle a^{2}\right\rangle \quad$ mean value of fiber radius squared

$\langle T$ mean value of normalized temperature

ance. The methods used for measuring these are well known. Results will be given and compared with theory.

The reported measurements were done with sound velocity perpendicular to the fibers of the glass wool.

The measurements will be compared with the theoretical predictions. A full report of the theory will be given in another paper at a later time. The purpose of measurements is to see if the physical assumptions of the theory are sufficient 
to describe accurately the acoustic properties of glass wool.

Early authors on measurement of the acoustic properties of sound absorbing material are Zwikker and Kosten (1949). They described many ways standing wave tubes may be used. In each case a sample of the material was placed in one end of a tube and in the other end was placed a sound source. Between the sound source and the sample was air. In this air a standing wave was generated. The ratio between the maximum and minimum of the sound pressure was measured. From this the numeric value of the impedance could be calculated. If one measures the distance from the sample to the first minimum of the standing wave, the phase of the sound sample impedance may be found.

The sample used for measurement by the tube method was placed on a rigid metal plate, which closed the tube. In the paper by Boker (1969) it is shown how one, from impedance measurements on samples of thickness 10 and $5 \mathrm{~cm}$, can compute the characteristic impedance. The wave vector for monochromatic waves may also be computed.

Hersh and Walker (1980) used a tube with a long sample, where reflections of waves in the sample could be neglected. They measured the pressure inside the sample with a very thin probe microphone. The distance from the surface of the sample to the microphone was measured, together with the sound pressure. From this they found the attenuation constant of the sample, that is, the imaginary part of the wave vector.

Allard et al. (1987) measured the impedance of a $1-\mathrm{m}^{2}$ blanket of glass wool placed on the asphalt of a large parking area. A wave of oblique incidence was generated by a loudspeaker several meters from the blanket. Sound pressure and velocity were measured by means of two microphones placed a little above the middle of the blanket. Allard et al. (1991) used a two-microphone method in an anechoic room with a rigid impervious floor to measure the impedance of the glass wool.

McIntosh et al. (1990) used a standing wave tube with several microphones that were used to measure the standing wave ratio. From the pressures detected by the microphones and their distances from the sample, the flow impedance and bulk modulus of the sample were computed from transfer function measurements. If properly implemented this method is accurate.

In most tube methods one measures the impedance of the sample including reflections of waves from a rigid back plate. In this paper we are interested in measuring the intrinsic properties of glass wool. We study the properties of glass wool itself independently of surfaces such as rigid back plate.

It was thought that it would be more accurate to use a more direct method than the one used by Boker (1969) to find the characteristic impedance and wave vector. It was therefore decided to use thick samples for the measurements of the characteristic impedance and the wave vector. The sound waves were attenuated in the glass wool. The wave reflected from the back of the sample could therefore not interfere with the incoming wave in the front of the sample, if the samples were thick enough. They were always so thick that these reflections could be neglected.
One can compute the compressibility and the flow resistance of the sample from measurements of the characteristic impedance and the wave vector. This was tried, but the results were very scattered due to experimental errors in the measurements of characteristic impedance and wave vector. Much more nearly accurate values were needed for comparison with theory. It was therefore decided to develop a new direct method of measuring the compressibility and flow resistance. This is presented in the following.

\section{THEORY}

A theory of compressibility and resistance of fiber materials has been worked out by the author. The details of the theory will be reported in another paper at a later time. Here some results will be given together with the assumptions of the theory. Only linear properties are considered, that is the sound pressure is assumed to be small.

\section{A. Compressibility}

Glass wool is regarded as a continuous medium for sound waves, because the distances between fibers are much smaller than the shortest audible sound wavelength. Sound is connected with flow and compression of air. For an infinitely small closed surface about a volume $V$, one defines the compressibility $C$ as

$$
C=\frac{d V}{V d p},
$$

where $d V$ is the change in volume, when the pressure change is $d P$.

When the air is compressed due to the sound waves, the temperature of the air between fibers increases. The temperature increase is diminished by the heat conduction from air to fibers. The temperature of the air is calculated by solving the differential equation for heat transport. From this one finds the compressibility. The compressibility divided by the adiabatic compressibility of air itself is

$$
C / C_{0}=\gamma-(\gamma-1)\langle T\rangle,
$$

$C_{0}$ is the adiabatic compressibility of air itself, $\gamma$ is the isothermal conıressibility divided by the adiabatic compressibility for air alone ( $\gamma=1.40$ for atmospheric air), and $(T\rangle$ is a mean "temperature" given by

$$
\langle T\rangle=\left(1-\frac{2 \tau^{-k} a H_{1}^{1}\left(k_{t} a\right)}{(k, b)^{2} H_{0}^{1}\left(k_{t} a\right)}\right)^{-1},
$$

where $a$ is the radius of the fibers, and $b^{2}$ is the mean area per fiber. The $H$ 's are Hankel functions of the first kind of zero and first order and $k_{\ell}$ is the thermal wave vector, which equals

$$
k_{f}=\sqrt{i \omega \rho_{0} c_{p} / \kappa},
$$

where $\omega$ is the cyclic frequency $=2 \pi \times$ frequency, $\rho_{0}$ is the mass density of ai-, $c_{p}$ is the heat capacity of air of constant pressure per mass unit, and $\kappa$ is the coefficient of thermal 
conductivity of air. In this paper we use the complex time factor,

$$
e^{-i \omega t}
$$

where $t$ is the time.

\section{B. Resistance}

Strictly speaking the resistance is a tensor because glass wool is anisotropic. However, if the pressure depends only on one space coordinate $x$, the resistance $R$ can be defined from

$$
-\frac{d p}{d x}=(R-i \omega \rho) u,
$$

where $\rho$ is the mass density of air and $R$ and $\rho$ are real quantities.

The sound wave causes the air to flow between the fibers of the glass wool. The friction between air and fibers gives resistance to the air flow. The velocity of the air between the fibers was calculated from the linearized Navier-Stokes equation. From the calculated velocity the acoustic resistance was found. For sound velocity perpendicular to the fibers, the low-frequency limit is approximately

$$
R=\frac{2 \pi \eta}{b^{2}\left[\ln (b / a)-\frac{3}{8}\right]} .
$$

\section{Wave vector and characteristic impedance}

The propagation of sound in homogeneous glass wool of infinite extent is considered. Plane monochromatic waves can propagate in the glass wool, but sound velocity and attenuation depend on the direction of propagation of the plane wave. Glass wool is anisotropic, because its fibers are almost all parallel with a plane. The pressure of a plane wave can be written

$$
p(x)=p_{0} e^{i k x},
$$

where $p(x)$ is the pressure at the coordinate $x, p_{0}$ is a constant, and $k$ is the complex wave vector. The real part of $k$ is the change of phase angle per meter, and the imaginary part is related to the attenuation of the sound wave. The wave vector can be computed from the resistance and compressibility,

$$
k=\sqrt{\left(\omega^{2} \rho+i \omega R\right) C} .
$$

We consider plane monochromatic sound waves in glass wool of infinite extent. The characteristic impedance $Z$ equals the complex pressure divided by the velocity,

$$
Z=p / u \text {. }
$$

The characteristic impedance is a complex number that depends on frequency. It depends also on the direction of propagation. The characteristic impedance of a plane traveling wave is calculated from the compressibility and resistance, and we have

$$
Z=\sqrt{(\omega \rho+i R) / \omega C} .
$$

\section{Assumptions of the theory}

The following is assumed in the theory: the fibers are parallel, the distances between fibers are random, all fibers have the same diameter, the fibers do not move.

These assumptions give results for the acoustic properties that are in accordance with all measurements. But they are not exactly fulfilled for real fiber materials.

Real glass wool consists of glass fibers in an irregular space lattice. The fibers are glued together with phenolformaldehyde resin. When seen in a microscope they are approximately parallel. In the bulk material they are to a high degree parallel to a fixed plane. It is therefore not unreasonable to assume they are parallel.

The fibers are irregularly placed in the wool. The theory assumes they are completely randomly distributed.

The theory shows that the acoustical properties of fiber material with fibers, which all have the same diameter, depend on the logarithm of the fiber diameter. The fiber diameters are not equal in real materials. But one may assume that the acoustical properties depend little on the diameter of the fibers. It is therefore reasonable to assume equal diameters of the fibers.

The mechanics of real glass wool interacting with the sound wave is very complex. It seems that for the density of glass wool used for acoustical purposes, the movement of the fibers may be neglected [see Allard et al. (1991)]. All the acoustical properties predicted from the present theory agree (within the experimental errors) with the measurements presented here.

\section{E. Values of the parameters of the theory}

The parameters of the theory are the average area per fiber and the mean diameter of the fibers.

The diameters were found by taking random samples and measuring the diameter with help of a microscope. The mean value was $6.8 \mu \mathrm{m}$ with a standard deviation of $2.7 \mu \mathrm{m}$ for glass wool with density $16 \mathrm{~kg} / \mathrm{m}^{3}$. Glass wool with a higher density of $40 \mathrm{~kg} / \mathrm{m}^{3}$ had the same mean fiber diameter.

The square root of the average area per fiber $b$ can be found from the mass density of the glass wool $\rho_{w}$, and the mass density of the glass in the fibers $\rho_{g}=2550 \mathrm{~kg} / \mathrm{m}^{3}$;

$$
b=\sqrt{\pi\left\langle a^{2}\right\rangle \rho_{g} / \rho_{w}},
$$

where $\left\langle a^{2}\right\rangle$ is the average of the square of the radius of the fibers. This was computed from the measured diameters of the fibers. The result was $b=75 \pm 5 \mu \mathrm{m}$ for glass wool with density of $16 \mathrm{~kg} / \mathrm{m}^{3}$ and $b=50 \pm 3 \mu \mathrm{m}$ for glass wool with density $40 \mathrm{~kg} / \mathrm{m}^{3}$.

\section{COMPRESSIBILITY}

\section{A. Measurement setup}

A tube was used as a resonator. Figure 1 shows the tube apparatus. The tube was made of PVC. It was circular with an internal diameter of $102 \mathrm{~mm}$, the wall was $4 \mathrm{~mm}$ thick. To the right in Fig. 1 was mounted a loudspeaker, the rear side 


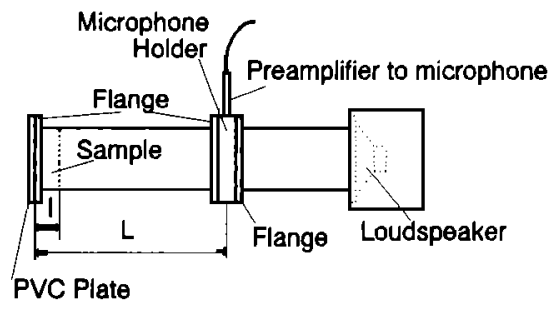

FIG. 1. The tube apparatus used to measure compressibility of glass wool. The internal diameter of the tube was $102 \mathrm{~mm}$.

of which was enclosed by a PVC tube. The loudspeaker was connected to a tube, on which was glued a flange seen in the middle of the figure. Next to this flange was placed a ringformed microphone holder, which was connected with screws to the flange of the left and right tube. A condenser microphone was used. Its membrane was placed flush with the internal surface of the tube. The left tube was closed with a $10 \mathrm{~mm}$ PVC plate fastened with screws to a flange on the tube. The glass wool sample was placed on the end plate inside the tube. The left tube could easily be replaced. Tubes of length from 0.2 to $3 \mathrm{~m}$ were used.

The frequency was always so small that only plane waves could propagate in the tube. At the highest frequency a tube with a cross section of $21 \times 21 \mathrm{~mm}^{2}$ was used. The electronic part of the measuring system is shown in Fig. 2. The filter was either a 1 - or $\frac{1}{3}$-octave filter.

\section{B. The idea of the measurement}

$A$ thin layer of glass wool was placed on the end plate of the tube. The distance from the closed end to the microphone was a quarter of the wavelength of sound in air at the frequency of measurement. The pressure at the microphone regarded as a function of frequency has a minimum at the resonance. The resonance frequency was measured for the tube both with and without the glass wool.

The air column may be regarded as mass-spring oscillator. The air at the microphone acts as the mass and the air at the closed end corresponds to the spring.

When there is glass wool in the tube, the spring is weaker than it is when the tube is empty. When air is compressed heat is generated and the temperature rises. But this temperature rise is small for the air between glass fibers, because heat is conducted from the air to the fibers. The heat conduction is fast because the distances between fibers are

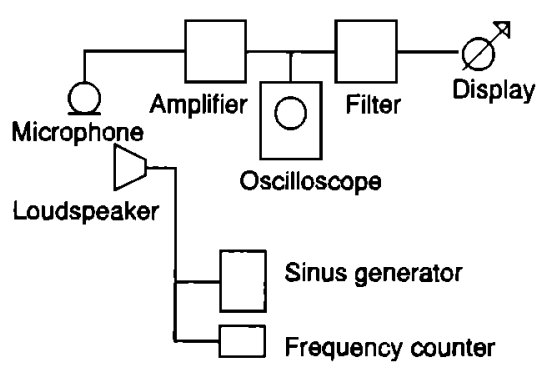

FIG. 2. The electronic setup used to measure compressibility and resistance. small. If the temperature rise is small the pressure rise is small, therefore the air spring is weak when it contains glass wool.

The resonance frequency is a little lower for the tube with glass wool than for the empty tube, because the weak spring gives a lower resonance frequency. If the shift in resonance frequency is measured, one may calculate the compressibility of the glass wool.

\section{Measurement procedure}

The length of the tube from the end plate to the middle of the microphone membrane was measured. The frequency of the pressure minimum of the tube without glass wool was measured. Actually, the two frequencies where the pressure was $3 \mathrm{~dB}$ over the pressure minimum where measured. The mean of these two frequencies was used as the resonance frequency, because this mean was more well defined than the frequency of the flat minimum.

The thickness of the sample was measured. The glass wool sample was placed on the end plate. The resonance frequency was measured.

The filter in Fig. 2 was necessary because the fundamental frequency of the sound pressure is suppressed by the resonance. Harmonics generated by the loudspeaker membrane were removed by the filter. These harmonics are not connected with nonlinearity of the sound waves.

The compression is nonlinear at high pressure. In order to check for nonlinearity, the frequencies were always measured with the following voltages on the loudspeaker 0.1 , 0.5 , and 1.0 V. A further check for linearity was done by measuring the pressure on the end plate before the actual measurements. This was done by replacing the end plate by a plate with a microphone holder. This is not shown in Fig. 1. The pressure was observed on the oscilloscope. The compression was linear for all voltages on the loudspeaker used in the following measurements.

\section{Calculation of compressibility}

The sound velocity generated by the loudspeaker at the microphone could be assumed to be constant in a narrow frequency band about the resonance frequency, because the sound pressure is very small in this frequency range. Therefore the resonance frequency was determined by the acoustic properties of the tube to the left of the microphone in Fig. 1.

The resonance frequency can be computed by Rayleigh's principle:

$$
\omega_{r}^{2}=\frac{\int_{V}(1 / \rho)(\nabla p)^{2} d \nu}{\int_{V} C_{f r} p^{2} d \nu},
$$

where $\omega_{r}$ is the cyclic resonance frequency. The integration is done over the volume of the tube between the end plate and the microphone. Here, $d \nu$ is the element of volume, $\rho$ is the mass density of air, and $C_{f r}$ is the real part of the compressibility in the tube $\left(C_{f r}=C_{0}\right.$ for points outside the glass wool). For points in the wool, $C_{f r}=C_{r}$, which is the real part of the compressibility of glass wool. It is assumed that the pressure in the tube with glass wool is equal to the pressure in an empty tube, 


$$
p=p_{0} \cos (\pi x / 2 L),
$$

$x$ is measured from the closed end of the tube along its axis and $L$ is the length of the tube from the closed end to the microphone.

The length of the glass wool sample was much shorter than the length of the tube. From this fact one can show that the real part of the compressibility of glass wool divided by the compressibility of air is

$$
\frac{C_{r}}{C_{0}}=\left(\frac{\omega_{r}^{2}}{\omega_{0}^{2}}-1\right) \frac{L}{2 l}+1,
$$

where $\omega_{0}$ is the cyclic resonance frequency of the empty tube, $\omega_{r}$ is the cyclic resonance frequency of the tube with glass wool, and $l$ is the length of the sample.

The formula (15), which serves to compute the real part of the compressibility from experimental data, is simple, but it is not sufficiently accurate. The pressure in formula (14) has too big a value in the glass wool, because the glass wool attenuates the sound. The real pressure is therefore different from the pressure in Eq. (14). The formula is accurate if one uses a very thin sample, but in this case, it is difficult to measure the shift of resonance frequency. At a frequency of $27 \mathrm{~Hz}$ the length of the tube was $3.13 \mathrm{~m}$, and the length of one glass wool sample was $0.30 \mathrm{~m}$. Here $C_{r} / C_{0}=1.29$ when computed from the above formulas. A more nearly precise computation is shown in the Appendix. It gave the value 1.38 .

The imaginary part of the compressibility may be found from the dissipation factor. It was found from measurement of the $3 \mathrm{~dB}$ width of the resonance curve and from the resonance frequency. It is

$$
d=\Delta f / f,
$$

where $d$ is the dissipation factor, $\Delta f$ is the 3 - $\mathrm{dB}$ bandwidth, and $f$ is the resonance frequency.

The dissipation factor equals the power loss divided by the energy of the resonator times the cyclic frequency. That is,

$$
d=P_{d} / E \omega_{r},
$$

where $P_{d}$ is the power loss and $E$ is the energy stored in the resonator

There are three contributions to the dissipation factor of the tube with glass wool: $d_{0}$ from the empty tube, $d_{C}$ from the imaginary part of the compressibility of the glass wool, and $d_{R}$ from the viscous boundary layer resistance of the glass wool. Thus we have

$$
d=d_{0}+d_{C}+d_{R} \text {. }
$$

The dissipation due to the compression of air in the glass wool can be written as

$$
d_{C}=\frac{\int_{V} C_{f i} p^{2} d \nu}{\int_{V} C_{f r} p^{2} d \nu}
$$

The integration is done over the volume in the tube between end plate and microphone. Here, $C_{f i}$ is the imaginary part of the compressibility, which is a function of position. Inside the glass wool, it equals $C_{i}$, which is the imaginary part of the compressibility. Outside the glass wools, it is zero. From formulas (14) and (19) one can show that the imaginary part of the compressibility divided by the compressibility of air is

$$
\frac{C_{i}}{C_{0}}=\left[\frac{L}{2 l}+\left(\frac{C_{r}}{C_{0}}-1\right)\right] d_{C},
$$

where $L$ is the length of the tube and $l$ is the length of the sample. The dissipation factor $d_{C}$ in formula (20) is due to the compressibility.

The dissipation factor due to the viscous resistance of the sample is needed. From Eq. (17) we get

$$
d_{R}=\frac{\int_{0}^{l} R u^{2} d x}{\omega \int_{0}^{L} \rho u^{2} d x},
$$

where $u$ is the velocity, which we assume is the same for the empty and the tube with glass wool. That is

$$
u=u_{0} \sin (\pi x / 2 L)
$$

where $u_{0}$ is a constant. When Eq. (22) is set into (21), one gets the dissipation factor from the resistance of the sample:

$$
d_{R}=\pi R l^{3} / 12 \rho_{0} L^{3} f \text {. }
$$

The value $R$ of the resistance was measured, and it is given in a following section of the paper. The dissipation factor due to the resistance was computed from (23); $d$ and $d_{0}$ were measured. From Eq. (18) one can compute the dissipation factor due to the compressibility. And from Eq. (20) one finds the imaginary part of the compressibility. The Appendix gives the corrections for the resistance of the glass wool in a more mathematical way.

The results of the measurements of the compressibility are shown in Fig. 3 for glass wool with a density of 16 $\mathrm{kg} / \mathrm{m}^{3}$. The thickness of the thin samples were found from the weight of the samples, their area, and the density of the glass wool.

\section{E. Comparison with theory}

The value of $b=100 \pm 5 \mu \mathrm{m}$ gave the best fit of theoretical results to the experimental data. From density measurements described in Sec. I E, it was found to be $75 \pm 5 \mu \mathrm{m}$.

Figure 3 shows the theoretical calculated curve together with the experimental points. The upper part of the figure shows the real part of the compressibility divided by the compressibility of air itself. At low frequencies the compressibility is high because the compression is isothermal, but at high frequencies it is low, because the compression is adiabatic.

The ratio between the compressibility at low frequencies and high ones is 1.40 , if the compression at low frequencies is isothermal. However, this is not the case for the light glass wool used here, because the heat capacity of the glass fibers is not infinitely great, compared to the heat capacity of air at constant pressure. The ratio between the heat capacity of glass and air in a volume of glass wool, equals 10 for glass wool density of $16 \mathrm{~kg} / \mathrm{m}^{3}$. From this one can compute the ratio between compressibility in the low- and high-frequency limit. This ratio is $\mathbf{1 . 3 6}$. The experimental data seems to be a little lower.

The lower part of Fig. 3 shows the imaginary part of the compressibility of glass wool divided by the compressibility 

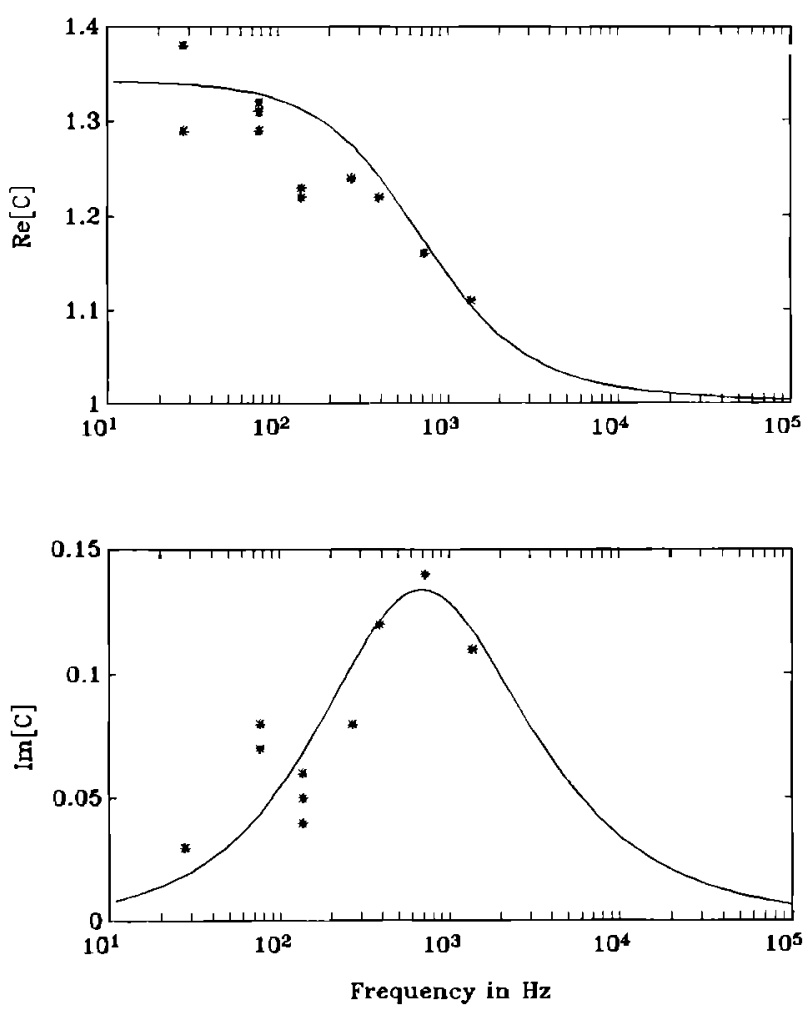

FIG. 3. The measured real and imaginary part of the compressibility of glass wool with a density of $16 \mathrm{~kg} / \mathrm{m}^{3}$ divided by the compressibility of air. The curve was calculated from a fiber radius of $3.4 \mu \mathrm{m}$ and an average distance between fibers of $100 \mu \mathrm{m}$.

of air itself. The imaginary part has a maximum at the transition frequency, where the compression goes from isothermal to adiabatic.

\section{RESISTANCE}

The resistance is an important property of glass wool. It determines, together with the compressibility, the sound velocity and attenuation. The resistance is defined in Eq. (6).

\section{A. Measurements setup}

The measurements were done with the tube shown in Fig. 1. The glass wool sample was placed across the tube close to the microphone at the left side in Fig. 1. At the frequency where the distance from microphone to the closed end of the tube was a quarter of a wavelength, the pressure was very low. The samples used at the high frequencies were thin, about $1 \mathrm{~mm}$, in order to have a resonance with a small dissipation factor. The samples were supported by thin steel rods that prevented them from moving.

The electronic system was the same as the one used for compressibility measurements, see Fig. 2.

\section{B. Measurements procedure}

The thickness of the sample was measured. The thickness of the thin samples used at high frequencies were found from their weight, area, and the density of the glass wool.

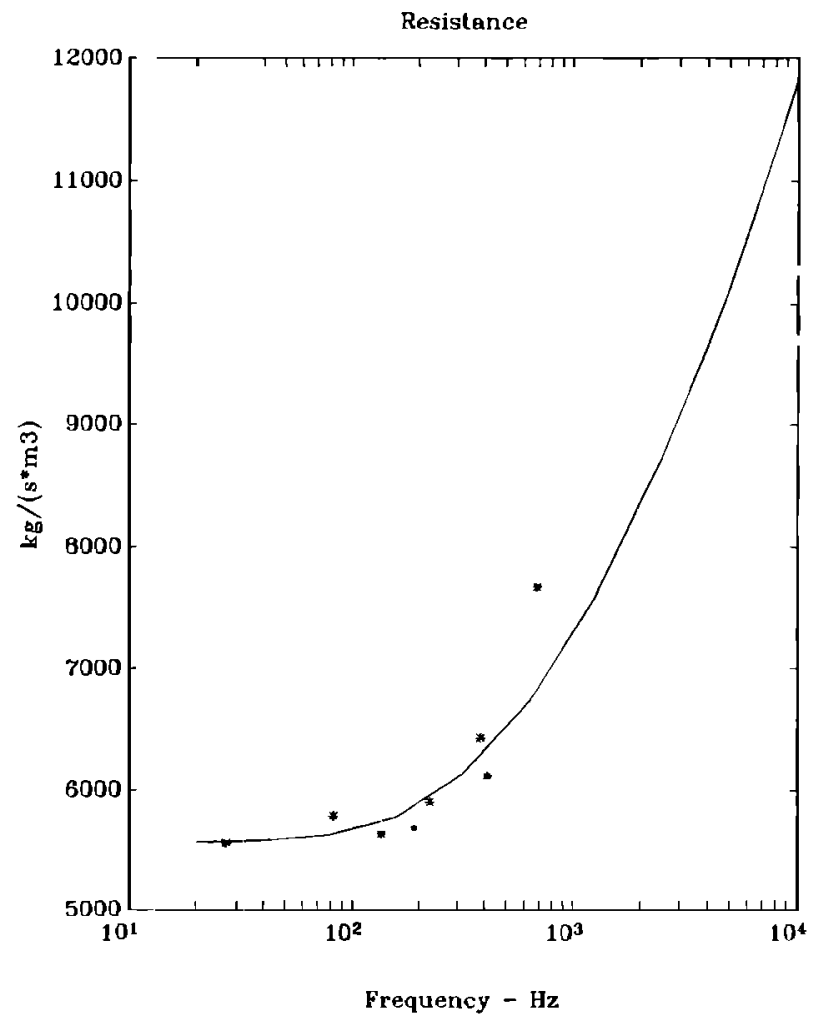

FIG. 4. The measured resistance of glass wool with a density of $16 \mathrm{~kg} / \mathrm{m}^{3}$. The sound velocity is perpendicular to the fibers. The curve was calculated from a fiber radius of $\mathbf{3 . 4} \mu \mathrm{m}$ and an average distance between fibers of 89 $\mu \mathrm{m}$.

The glass wool was placed in the tube. The resonance frequency and the 3-dB width of the resonance curve was measured. From this one can calculate the resistance.

The shift of the resonance frequency from the empty tube to the one with glass wool can in principle give $\rho$ in formula (21). But it was not possible to detect such a frequency shift because the resonance curve was too broad.

\section{Calculation of resistance}

The dissipation factor for the resonator was calculated from Eq. (16). It was to be calculated from the measured data.

The dissipation factor can be found from Eq. (21). This is done by setting the velocity from Eq. (22) into (21). One gets

$$
R=L \omega \rho_{0} d / 2 l \text {. }
$$

The results of the measurements are shown in Fig. 4. It shows the real part of the resistance. The continuous curve was calculated from theory. At low frequencies the real part of the resistance is constant. The resistance is proportional to the friction force on the fibers for a given mean velocity of air between the fibers. The force of friction is proportional to the gradient of the air velocity at the fiber. The gradient does not depend on frequency, if it is low. Therefore the resistance does not depend on frequency. At high frequencies, however, the friction takes place in a thin boundary layer close to the fibers. In this boundary layer, the gradient of the velocity is higher than it is in the low-frequency case. Thus resistance rises with frequency. 


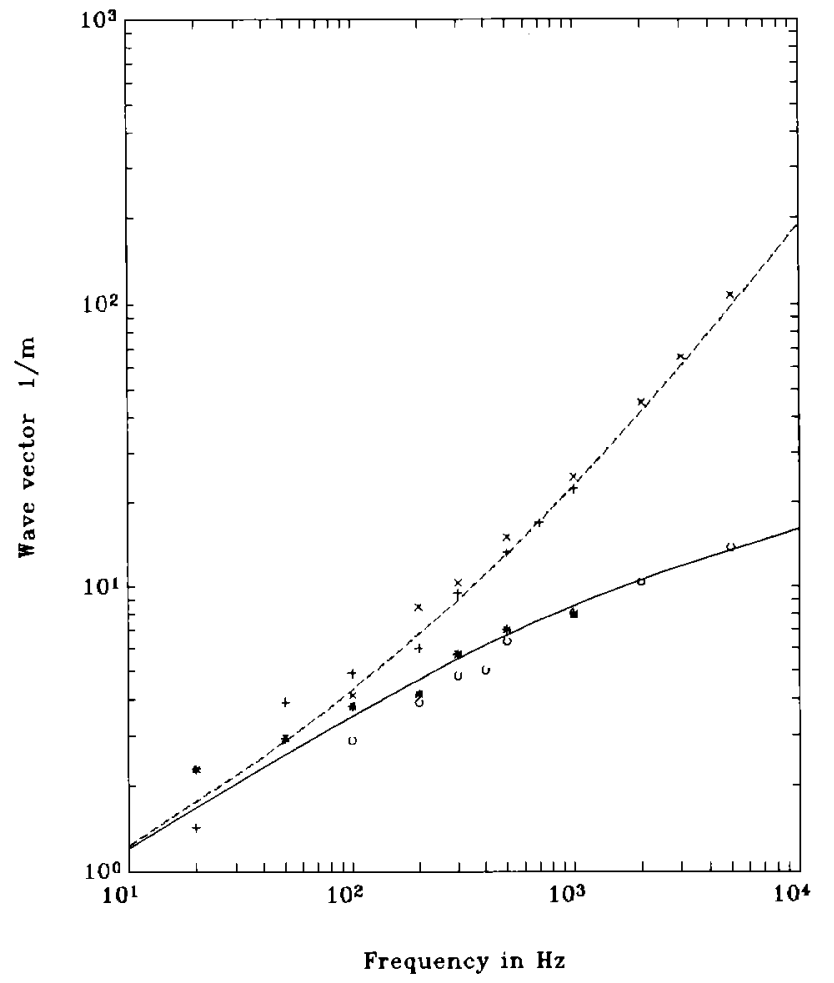

FIG. 5. The measured wave vector for a density of $16 \mathrm{~kg} / \mathrm{m}^{3}$. The sound velocity is perpendicular to the fibers: ${ }^{*}$ points are attenuation; + are phase, both measured with the tube; $O$ are attenuation; $X$ are phase, both measured in anechoic room. The curves were calculated for fibers of radius $3.4 \mu \mathrm{m}$, and an average distance between fibers of $95 \mu \mathrm{m}$.

\section{Agreement with theory}

The continuous curve in Fig. 4 was computed from the theory. The radius of the fibers was assumed to be $3.4 \mu \mathrm{m}$. It was measured as described in Sec. IE and $b=89 \pm 2 \mu \mathrm{m}$ gave the best fit to the experimental data. The resistance is sensitive to this distance. The quantity $b=75 \pm 5 \mu \mathrm{m}$ was found from the mass density. This is not far from the value from the measurements of resistance. One can conclude that the agreement between measurement and experiment is good.

\section{ATTENUATION AND PHASE SHIFT OF PLANE TRAVELING WAVES}

The definition of the wave vector is given in Eq. (8). We present in this section results of measurements of the wave vector for glass wool with two densities. Two measurement methods were used. A tube method and measurement in an anechoic room. The two methods were used in order to get more reliable data by measuring the same physical object in different ways. Also, as the two methods do not have the same frequency range, they supplement each other.

The measurements are compared with values theoretically computed from densities and diameters of fibers.

\section{A. Tube measurements}

A thick layer of glass wool was placed in a PVC tube with an internal diameter of $102 \mathrm{~mm}$. Sound waves were generated by a loudspeaker mounted in the tube. It was fed

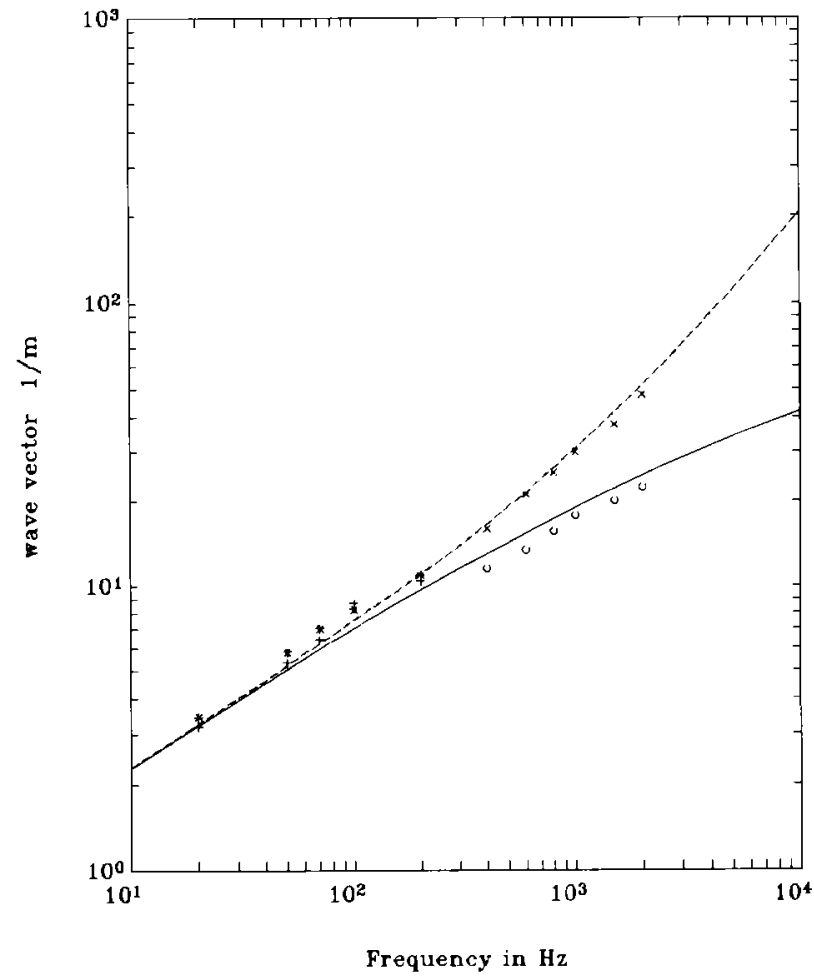

FIG. 6. The measured wave vector for a density of $40 \mathrm{~kg} / \mathrm{m}^{3}$. The sound velocity is perpendicular to the fibers: * points are attenuation; + are phase, both measured with the tube; $O$ are attenuation; $x$ are phase, both measured in anechoic room. The curves were calculated for fibers of radius $3.4 \mu \mathrm{m}$, and an average distance between fibers of $55 \mu \mathrm{m}$.

with sine voltage. The sound pressure in the glass wool was measured by a microphone mounted flush with the wall of the tube. The microphone could be placed in several holes in the wall of the tube. The holes not in use were closed airtight. The pressure was measured at different distances from the surface of the sample. The frequency and the voltage to the loudspeaker were constant. The glass wool sample was so thick that waves reflected from the end of it could be neglected. For the lowest frequency measurements the thickness was $0.5 \mathrm{~m}$.

The sound pressure in $\mathrm{dB}$ was plotted as a function of the distance from the loudspeaker, this is not shown in the figures. The points should ideally lie in a straight line, if the sample is thick enough. A line was drawn through the points. The slope of this line gave the imaginary part of the wave vector. Hersh and Walker (1980) used the same the procedure.

The real part of the wave vector was measured in a similar way. A phase meter was connected to the microphone output voltages and the loudspeaker voltage. The phase difference between loudspeaker voltages and microphone voltages was measured for the same positions of the microphone that was used for the attenuation measurements. The phase was plotted versus the distance from the loudspeaker. The points should lie on a straight line. A line was drawn through the points. The slope of this line gave the real part of the wave vector. 


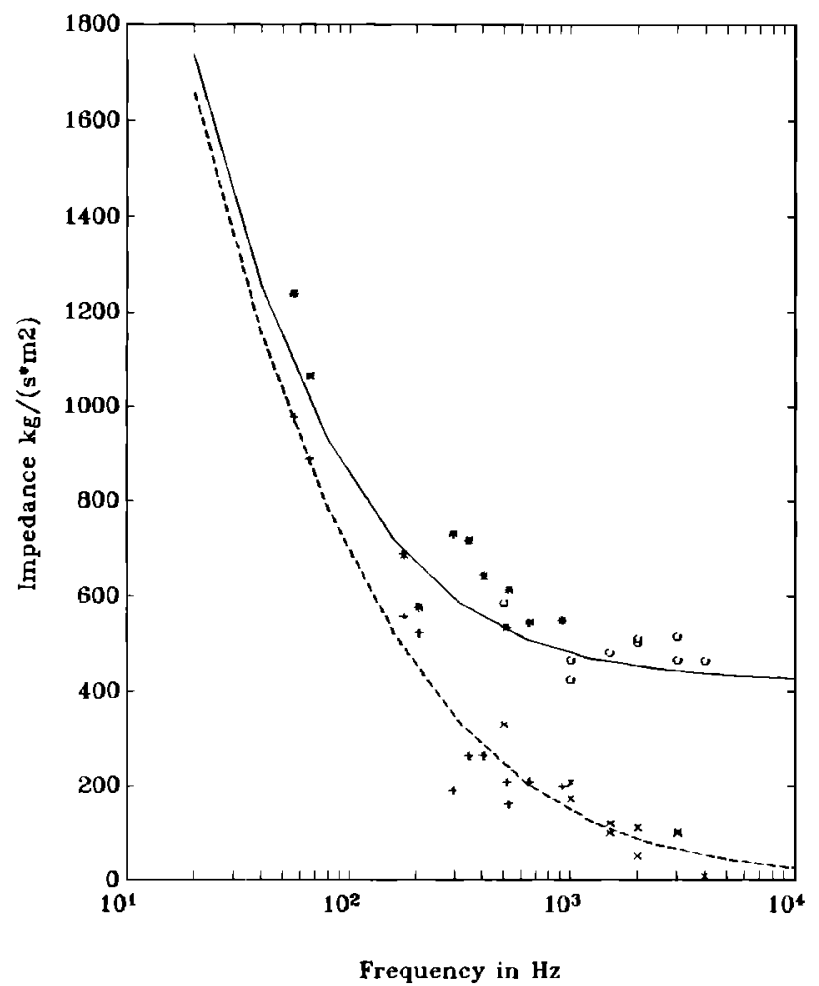

FIG. 7. The measured impedance for a density of $16 \mathrm{~kg} / \mathrm{m}^{3}$. The sound velocity is perpendicular to the fibers: * points are real parts; + are imaginary ones, both measured with the 102 -mm-diam tube; $O$ are real parts; $x$ are imaginary ones, both measured with a tube with cross section $21 \times 21$ $\mathrm{mm}^{2}$. The curves were calculated for fibers of radius $3.4 \mu \mathrm{m}$, and an average distance between fibers of $80 \mu \mathrm{m}$.

Figures 5 and 6 show the real and imaginary part of the wave vector for two densities of glass wool.

\section{B. Measurements in anechoic room}

Measurements of the wave vector were also done in an anechoic room. The glass wool samples were four slabs with dimensions $0.1 \times 0.6 \times 0.9 \mathrm{~m}^{3}$. They were placed in a pile that attenuated the sound waves and prevented interference from waves in the wool reflected from the rear side of the pile. In order to prevent interference from waves from the side of the pile, it was placed in a box of $20-\mathrm{mm}$ plywood sheets open on one side that faced a loudspeaker mounted above the box. The attenuation and phase were measured in the same way as with the tube measurements. The results are shown in Figs. 5 and 6.

\section{Agreement with theory}

The continuous curves in Figs. 5 and 6 were computed from the theory given earlier in this paper.

The diameters of the fibers were known. The value of $b$ that gave the best fit between theory and experiment was $95 \pm 5$ and $55 \pm 5 \mu \mathrm{m}$. The corresponding values found from the mass density are $75 \pm 5$ and $50 \pm 3$. One can conclude that the agreement between experiment and theory is sufficiently good to use the theory to predict the wave vector from fiber diameters and density.

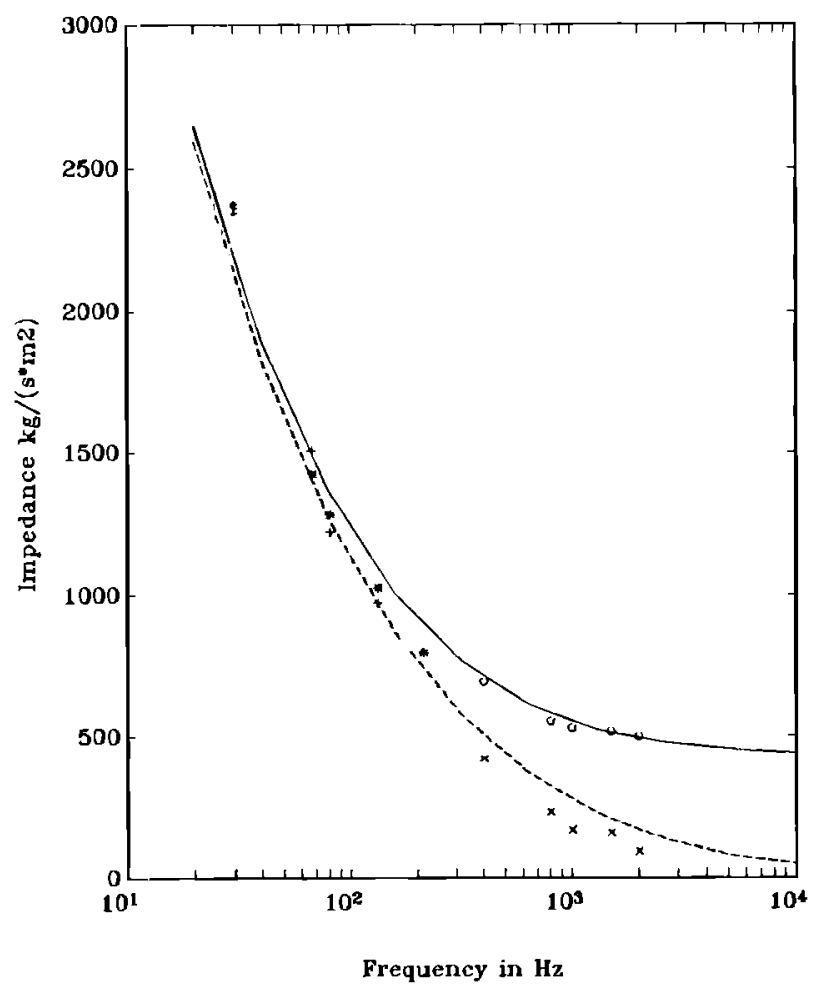

FIG. 8. The measured impedance for a density of $40 \mathrm{~kg} / \mathrm{m}^{3}$. The sound velocity is perpendicular to the tibers: * points are real parts; + are imaginary ones, both measured with the tube; $O$ are real parts; $X$ are imaginary ones, both measured in anechoic room. The curves were calculated for fibers of radius $3.4 \mu \mathrm{m}$, and an average distance between fibers of $55 \mu \mathrm{m}$.

\section{CHARACTERISTIC IMPEDANCE}

The characteristic impedance is defined in Eq. (10). In the measurements reported here, the sound velocity was perpendicular to the fbers of the glass wool. The measurements were done in a tube and in an anechoic room.

\section{A. Tube measurements}

The tubes and samples used for wave vector measurement were also used for impedance measurements. The pressure of the standing wave in front of the glass wool sample was measured at two distances from the sample by a microphone mounted flush with the wall of the tube. The distances of the microphones from the sample were measured. From this the impedance of the glass wool sample was calculated in the usual way [see McIntosh et al. (1990)].

The samples were thick so that waves reflected from the back of the sample were attenuated and could not interfere with the sound wave entering the sample. Thus the measured impedance equals the characteristic impedance of the glass wool.

The results are shown in Fig. 7 for glass wool with a density of $16 \mathrm{~kg} / \mathrm{m}^{3}$, and in Fig. 8 for a density of $40 \mathrm{~kg} / \mathrm{m}^{3}$.

\section{B. Measurementsi in anechoic room}

Impedance measurements were done in an anechoic room, with the same samples as were used for wave vector measurements. The impedance was measured with the two- 
TABLE I. Average distance between fibers delermined from mass density of wool, compressibility, resistance, wave vector, and impedance. The mean diameter of fibers was measured to $6.8 \mu \mathrm{m}$.

\begin{tabular}{lccccc}
\hline \hline $\begin{array}{l}\text { Density } \\
\text { of wool } \\
\mathrm{kg} / \mathrm{m}^{3}\end{array}$ & $\begin{array}{c}\text { Density } \\
\mu \mathrm{m}\end{array}$ & $\begin{array}{c}\text { Compressibility } \\
\mu \mathrm{m}\end{array}$ & $\begin{array}{c}\text { Resistance } \\
\mu \mathrm{m}\end{array}$ & $\begin{array}{c}\text { Wave vector } \\
\mu \mathrm{m}\end{array}$ & $\begin{array}{c}\text { Impedance } \\
\mu \mathrm{m}\end{array}$ \\
\hline 16 & $75 \pm 5$ & $100 \pm 5$ & $89 \pm 2$ & $95 \pm 5$ & $80 \pm 5$ \\
40 & $50 \pm 3$ & $\ldots$ & $\cdots$ & $55 \pm 5$ & $55 \pm 3$ \\
\hline \hline
\end{tabular}

microphone method in the way it was done with the tube. The results are shown in Fig. 8.

\section{Agreement with theory}

The continuous curves in Figs. 7 and 8 were computed from the theory in Sec. I. The diametèrs of the fibers were known. The values of $b$ that gave the best fit between theory and experiment were $80 \pm 5$ and $55 \pm 3 \mu \mathrm{m}$. This should be compared with the values found from mass density $75 \pm 5$ and $50 \pm 3 \mu \mathrm{m}$. One can conclude that the agreement between experiment and theory is sufficiently good to use the theory to predict the wave vector from fiber diameters and density.

\section{CONCLUSION}

A new accurate method for measuring the resistance and compressibility of acoustic absorbers has been developed. The method was used on glass wool and the results were compared by theoretical computed values. Good agreement was found.

Measurements of characteristic impedance, attenuation, and phase of monochromatic plane traveling sound waves in glass wool were also reported. The measurements were compared to theoretical results. Good agreement between experimental results and theoretical ones was obtained, see Table I. In the theory it is assumed that the fibers are parallel, the distances between fibers are random, all fibers have the same diameter, and the fibers do not move. These assumptions are sufficient to compute the acoustical properties of glass wool with good accuracy. They are adequate for predicting these properties in practical use of glass wool for acoustic purposes.

\section{APPENDIX: CORRECTIONS FOR COMPRESSIBILITY MEASUREMENTS}

The sound pressure in the glass wool placed in the quarter wavelength resonator was smaller than the pressure in the empty tube. This fact influences the computation of the real part of the compressibility. The resistance of the glass wool gives a dissipation of energy that must be taken into account when one computes the imaginary part of the compressibility from the measured data. The necessary corrections will be shown in this Appendix.

We start by writing the wave equations for the sound in the tube. The momentum equation is

$$
-\nabla p=\left(R_{f}-i \omega \rho\right) \bar{u},
$$

where $R_{f}$ is a function of place. It is zero outside the sample. The equation of continuity is

$$
-\nabla \cdot \bar{u}=C_{f}(-i \omega) p,
$$

where $C_{f}$ is a function of place. Inside the glass wool it equals the complex compressibility of the wools; outside it equals the compressibility of air itself, $C_{0}$. From Eq. (A1) compute the velocity

$$
\bar{u}=\frac{\nabla p}{i \omega \rho-R_{f}},
$$

Put Eq. (A3) into (A2)

$$
\nabla \cdot\left(\frac{\nabla p}{i \omega \rho-R_{f}}\right)=i \omega C_{f} p \text {. }
$$

This equation together with the boundary conditions defines an eigenvalue problem. The boundary conditions are: the velocity is zero on the end wall of the tube and the pressure is zero at the microphone. The eigenfrequency and eigenfunction of this equation are complex.

Equation (A4) is multiplied by the complex conjugate of the pressure

$$
p^{*} \nabla \cdot\left(\frac{\nabla p}{i \omega \rho-R_{f}}\right)=i \omega C_{f} p^{*} p
$$

This equation is integrated over the resonator volume. Partial integration is performed by use of Gauss' theorem for vectors. The term on the surface of the resonator is zero due to the boundary conditions. One gets the following equation for the complex resonance frequency $\omega_{c}$ :

$$
\int_{V} \frac{|\nabla p|^{2}}{R_{f}-i \omega_{c} \rho} d \nu=i \omega_{c} \int_{V} C_{f}|p|^{2} d \nu
$$

The pressure can be computed exactly. But the exact solution is too complicated to be useful.

The pressure outside the sample is

$$
p=p_{0} \cos (\pi x / 2 L), \text { for } l<x<L .
$$

The pressure in the glass wool can be expressed by the wave vector

$$
k=\sqrt{\left(\omega_{c}^{2} \rho+i \omega_{c} R_{f}\right) C} .
$$

In the glass wool, the pressure is

$$
p=p_{1} \cos (k x), \text { for } 0<x<l,
$$

where

$$
p_{1}=\frac{p_{0} \cos (\pi l / 2 L)}{\cos (k l)} .
$$

This value makes the pressure inside the glass wool continuous with the pressure outside the glass wool.

The velocity in the tube with glass wool is taken to be

$$
u=\frac{\pi p_{0}}{i \omega \rho_{0} 2 L} \sin \left(\frac{\pi x}{2 L}\right), \text { for } 0<x<L .
$$

The gradient of the pressure was found from Eqs. (A1) and (A11). This gradient and the pressure from Eqs. (A9) and 
(A10) were set into Eq. (A6) in order to calculate the compressibility.

Allard, J. F., and Bourdier, R., and l'Esperance, A. (1987). “Anisotropy effect in glass wool on normal impedance in oblique incidence," J. Sound Vib. 114 (2), 233-238.

Allard, J. F., and Depollier, C., and Guignourd, P., and Rebillard, P. (1991). "Effect of a resonance of the frame on the surface impedance of glass wool of high density and stiffness," J. Acoust. Soc. Am. 89, 999-1001.
Boker, A. (1969). "Attenuation of sound in lined ducts," J. Sound Vib. 10 (3), 390-403.

Hersh, A. S., and Walker, B. (1980). "Acoustical behaviour of bomogeneous bulk materivls," ALAA-80-0986 paper of Aeroacoustics Conference.

McIntosh, J. D., and Zuroski, M. T., and Lambert, R. F. (1990). "Standing wave apparatus for measuring fundamental properties of acoustic materials in air," J. Acoust. Soc. Am. 88, 1929-1938.

Zwikker, C., and Kosten, C. W. (1949). Sound Absorbing Materials (Elsevier, Amsterdam). 